\title{
Metachronous multiple chest wall osseous hemangiomas
}

\author{
Ke-Cheng Chen, MD, ${ }^{a}$ Chen-Tu Wu, MD, ${ }^{b}$ Chien-Te Pan, MD, and \\ Yung-Chie Lee, MD, PhD, ${ }^{a}$ Taipei, Taiwan
}

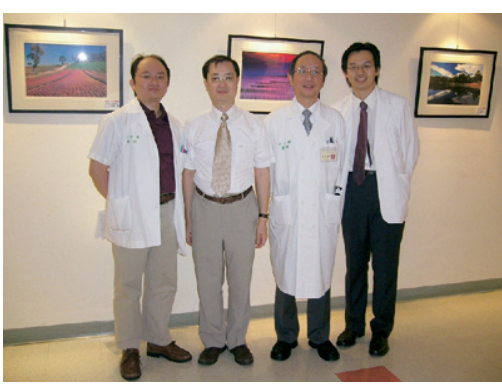

hest wall osseous hemangioma is a rare disease entity, accounting for only $0.7 \%$ of all hemangiomas. ${ }^{1}$ It may arise in the rib, clavicle, or sternum, but no metachronous multiple masses have ever been reported. We describe the case of a middle-aged woman with 4 metachronous chest wall osseous hemangiomas occurring in the sterum, clavicle, and bilateral ribs during 13 years of treatment and follow-up. To the best of our knowledge, this is the first case report of chest wall osseous hemangioma with this unique presentation ever published.

\section{Clinical Summary}

A 49-year-old previously healthy woman was admitted because of a palpable painless mass in the precordial area, which she found herself in 1992. There was no history of chest wall trauma and her family history was unremarkable. The plain chest film and computed tomography (CT) showed a large, well-enhanced expansile sternal lesion measuring $18 \times 7 \mathrm{~cm}$ (Figure 1, A). Diagnosis and treatment consisted in en bloc resection of the tumor followed by reconstruction with three titanium plates. Microscopic examination disclosed aggregates of closely packed, thin-walled, blood-filled capillaries separated by scant connective tissue stroma in the bone trabeculae, indicating a hemangioma. She recovered very well and was followed up at our hospital regularly. However, after 2 years, a mass was discerned at the left clavicular head by a chest $\mathrm{x}$-ray film.

From the Division of Thoracic Surgery, Department of Surgery, ${ }^{a}$ and Department of Pathology, ${ }^{\mathrm{b}}$ National Taiwan University Hospital and National Taiwan University College of Medicine, Taipei, Taiwan.

Received for publication Sept 27, 2006; accepted for publication Oct 24, 2006.

Address for reprints: Yung-Chie Lee, MD, PhD, Professor, Department of Surgery, National Taiwan University Hospital, 7, Chung-Shan South Rd, Taipei, Taiwan 100 (E-mail: wuj@ha.mc.ntu.edu.tw).

J Thorac Cardiovasc Surg 2007;133:838-9

$0022-5223 / \$ 32.00$

Copyright $\odot 2007$ by The American Association for Thoracic Surgery doi:10.1016/j.jtcvs.2006.10.058

Chest CT showed a clavicular tumor with bony disruption and cortex thinning (Figure 1, B).

A second operation for resection of tumor from the left clavicle was performed. The pathologic diagnosis was an osseous hemangioma again. She tolerated the surgery well and was discharged soon. Unfortunately, the follow-up image in 1999, when she was 56 , revealed another tumor on the left fifth rib (Figure 2, A). She then underwent a third operation, segmental rib resection. The pathologic examination again showed an osseous hemangioma. She had a disease-free interval of 6 years after the third operation. In April 2005, she was admitted again because an image study showed an expansile, well-enhanced mass at right eighth rib (Figure $2, B)$. A segmental rib resection was performed, and the diagnosis was again osseous hemangioma (Figure 2, $A$ and $B$ ). The postoperative course was smooth, and she was discharged 4 days after the operation. In her latest outpatient follow-up, 24 months after the most recent operation, she was well with no symptoms and no evidence of disease.

\section{Discussion}

Hemangioma is defined as a neoplastic entity that arises from blood vessels. Although skin is by far the most common primary site of hemangioma, any organ, including the bone, can be affected. Hemangioma of the bone is an uncommon benign vascular tumor and accounts for about $1 \%$ of all bone tumors in some series in the literature..$^{2,3}$ Although most of these cases occur in the skull or vertebral bodies as congenital lesions, some have been reported to originate from the chest wall, including the ribs, clavicles, and sternum. ${ }^{4,5}$ However, chest wall osseous hemangiomas are very uncommon, accounting for only $0.7 \%$ of all hemangiomas. ${ }^{1}$

Chest wall osseous hemangioma usually presents as a painless, large tumor and is therefore discovered by conventional radiography. Radiographically, bony hemangiomas usually present as radiolucent, well-demarcated defects. They expand and thin the cortex in flat bones, but complete cortical disruption is not

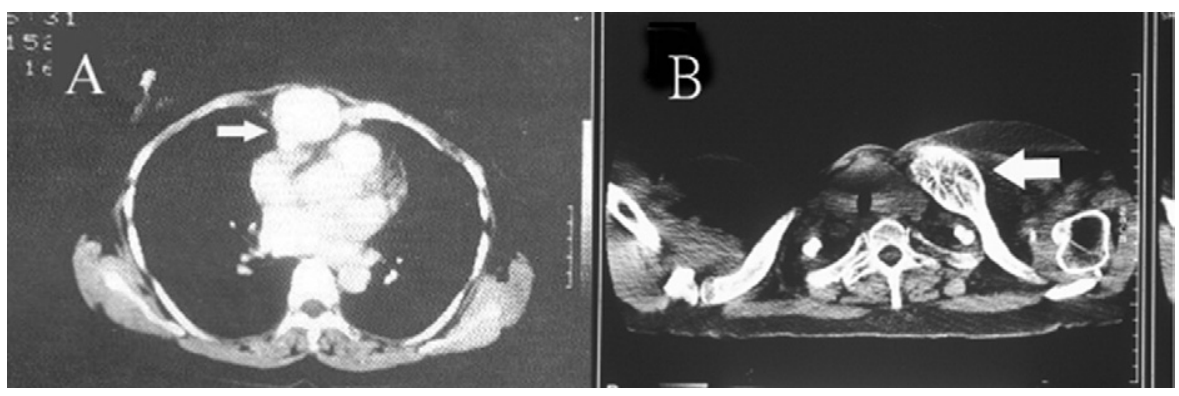

Figure 1. A, Initial chest CT, when the woman was 49 years old, showed a large, well-enhanced sternal tumor (arrow). B, Second chest CT, when she was 51, revealed a left clavicular tumor with bone medulla disruption and cortex thinning (arrow). CT, computed tomography. 


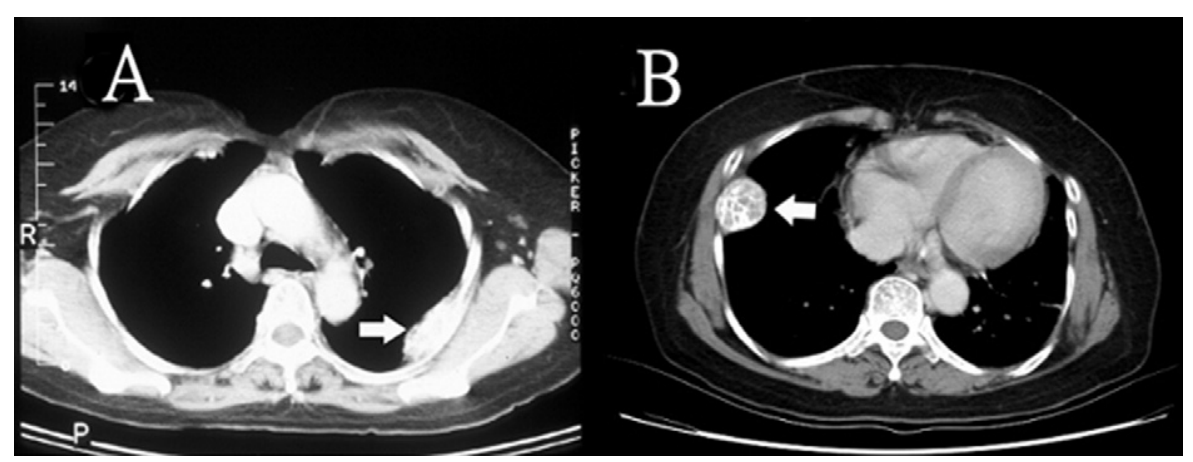

Figure 2. A, Third CT, when she was $\mathbf{5 6}$ years old, showed an expansile tumor at the posterolateral part of the fifth rib (arrow). B, Fourth CT, when she was 62 years old, showed a localized expansive growth of the tumor (arrow) projecting toward the thoracic cavity located at eighth rib. CT, computed tomography.

present. ${ }^{3}$ The characteristic radiographic findings reflect the formation of reactive spicule produced by the lesion. These findings are generally described as "sunburst appearance" for skull hemangioma, "corduroy-like appearance" for vertebra hemangioma, and "honeycomb appearance" for chest wall osseous hemangioma. ${ }^{3}$ The size and the extent of cortical destruction can be more clearly demonstrated by CT and magnetic resonance imaging. However, before surgery, it is sometimes difficult to establish the correct diagnosis and to differentiate these tumors from other malignant bone lesions that would require extensive tumor resection. In many cases, wide excision is necessary to provide adequate tissue for exact histologic diagnosis. For chest wall osseous hemangioma, surgical resection of the affected region is the treatment of choice. Although it is an extremely uncommon tumor that has been found incidentally, it should be considered in the differential diagnosis of chest wall tumors, especially in asymptomatic patients.

In summary, our patient initially had a sternal hemangioma that was widely excised, followed by reconstruction with titanium plates. However, multiple bony hemangiomas developed in the chest wall metachronously, including the left clavicle, left fifth rib, and right eighth rib sequentially in the following 13 years. To the best of our knowledge, this is the first case report of chest wall osseous hemangioma with this unique manifestation ever published.

\section{References}

1. Watson WL, McCarty WD. Blood and lymph vessel tumor. A report of 1056 cases. Surg Gynecol Obstet. 1940;71:569-88.

2. Dahlin DC. Bone tumors: general aspect and data on 6,221 cases. Springfield [IL]: Charles C Thomas; 1981. p. 137-48.

3. Dorfman H, Czerniak B. Vascular lesions. In: Bone tumors. St Louis: Mosby; 1997. p. 29-814.

4. Okumura T, Asamura H, Kondo H, Matsuno Y, Tsuchiya R. Hemangioma of the rib: a case report. Jpn J Clin Oncol. 2000;30:354-7.

5. Shimizu K, Yamashita Y, Hihara J, Seto Y, Toge T. Cavernous hemangioma of the rib. Ann Thorac Surg. 2002;74:932-4. 